\title{
Réflexions sur le concept de droit à partir de quelques cas limites
}

\section{Baudouin Dupret}

Centre d'étude des mouvements sociaux - Institut Marcel Mauss (CEMS-IMM), École des hautes études en sciences sociales, 190-198 avenue de France, F-75013 Paris.

<baudouin.dupret@ehess.fr>

\section{À propos de...}

- Bras Jean-Philippe (dir.), Faire l'histoire du droit colonial. Cinquante ans après l'indépendance de l'Algérie, Paris : Karthala, 2015, 322 p.

- Dresch Paul et Scheele Judith (eds.), Legalism: Rules and Categories, Oxford : Oxford University Press, 2015, 320 p.

- Dresch Paul et Skoda Hannah (eds.), Legalism: Anthropology and History, Oxford : Oxford University Press, 2012, 368 p.

- Fekry Ibrahim Ahmed, Pragmatism in Islamic Law: A Social and Intellectual History, Syracuse : Syracuse University Press, 2015, 320 p.

- Kuran Timur, The Long Divergence: How Islamic Law Held Back the Middle East, Princeton : Princeton University Press, 2011, 405 p.

Cinq ouvrages sont ici l'occasion de revenir sur la question du concept de droit et sur sa pertinence dans l'analyse de la normativité en général. Ils nous aident à jeter les bases d'une clarification conceptuelle à partir de situations limites, hors des seuls temps et espace du droit occidental moderne.

\section{Structures d'opportunité}

Dans The Long Divergence, Timur Kuran peint une grande fresque historique des relations du droit à l'économie en Méditerranée pour tenir l'argument que c'est le droit islamique qui, structurellement, a empêché le développement économique des sociétés d'islam. Partant de l'affirmation que «les institutions économiques prémodernes du Proche-Orient étaient largement fondées sur le droit dominant de la région, à savoir le droit islamique » (p. x), il s'attache à montrer comment la caractéristique essentielle d'une économie moderne a fait défaut à cette région du monde. II s'agit de la capacité à «mobiliser les ressources productives à une très grande échelle, à l'intérieur d'entreprises privées de long terme et la prestation de services par des entités durables et capables de transformation » (p. 5). Il situe le tournant au 
XVIII ${ }^{\mathrm{e}}$ siècle, c'est-à-dire au moment où les institutions liées à l'islam, qui fournissaient jusqu'alors des avantages statiques, sont devenues dynamiquement désavantageuses. Reprenant à son compte le concept de « dépendance trajectorielle » (path dependence), il entend par là que des institutions conçues pour assurer la prospérité, garantir le lien familial et promouvoir la stabilité politique peuvent finir par s'avérer dysfonctionnelles, sans qu'il ne soit possible de sortir de la piste historiquement tracée. L'idée est aussi que le développement endogène d'une société peut être poursuivi jusqu'aux limites de ce que ses ressources intellectuelles propres lui permettent, mais qu'il n'est possible de dépasser ces limites qu'à la condition de procéder à une révolution qui, en recourant à des ressources qui lui sont extérieures, bouleverse les fondements de cette société (p. 9).

À la voie de la modernisation suivie en terre d'islam, il eut sans doute été possible de substituer une alternative radicale, celle de l'importation massive de règles, dans le domaine du mariage et de l'héritage particulièrement, libérant ces sociétés de contraintes fortes pour la croissance et la longévité des entreprises commerciales, mais cela ne se pouvait pas, structurellement, dès lors que différentes règles dans ce domaine font l'objet de dispositions coraniques explicites (p. 93). Différents obstacles très concrets se sont élevés, dans l'univers musulman, à l'apparition de ressources juridiques favorisant le développement du grand capitalisme, parmi lesquels, en vrac, le manque de besoin d'innovation, la stagnation du régime des fondations (waqf), le rôle de l'État, le rôle négatif de l'affermage fiscal, l'absence de sociétés familiales, l'inexistence de la notion de personnalité morale. Ce n'est évidemment pas que le droit islamique serait immuable de manière inhérente, non plus que ses interdits ne pourraient être contournés par des esprits inventifs. L'exemple de l'intérêt est parlant, de ce point de vue, qui nous montre que de multiples stratégies furent inventées pour contourner la lettre de la Loi religieuse (p. 147). L'argument de Timur Kuran est plutôt structurel et dynamique : des institutions sont établies à partir des ressources disponibles, qui vont ensuite se cristalliser et s'inscrire profondément dans la structure sociale ; ces institutions, sans doute vertueuses à l'origine, peuvent se transformer en freins au développement économique et social par la suite, et de la sorte générer un cercle vicieux. Ainsi en va-t-il du principe d'élection de droit accordé aux nonmusulmans en vertu du système de protection (dhimma). C'est-ce qu'on appelle le mécanisme Tiebout, en vertu duquel la circulation inter-juridictionnelle des individus renforce l'efficacité sociale d'une institution, alors que, paradoxalement, la volonté de réforme intra-institutionnelle porte préjudice à la juridiction qui l'entreprend.

Par ailleurs, certaines institutions islamiques se sont avérées inhibitrices d'un développement vers le système bancaire moderne. L'argument conservateur consiste d'habitude à dénoncer l'institution bancaire en tant qu'elle serait la preuve d'une occidentalisation destructrice et qu'elle serait l'instrument impérialiste de l'asservissement de l'Occident, mais la question demeure de savoir pourquoi cette institution s'est imposée. Pour T. Kuran, c'était la réponse naturelle aux avancées structurelles de l'Occident, qui n'avaient pu se produire en contexte musulman du fait des obstacles posés par le droit islamique (p. 166). Autrement dit, le mimétisme juridique et judiciaire n'est pas tant un effet de l'impérialisme que celui des avantages compa- 
ratifs de la technologie institutionnelle occidentale. L'exemple des capitulations est, à cet égard, très parlant, dès lors que, d'un système de privilèges et facilités réciproques, il s'est transformé en un outil de discriminations patentes et a fini par aboutir au système des juridictions mixtes (p. 222). Si le constat de la discrimination est correct, la question est ici aussi de savoir ce que pouvaient trouver les acteurs économiques dans la mise en place de ce droit et de ces juridictions, sinon le remède aux dysfonctionnements, directs ou indirects, du droit islamique (favoritisme local, manque de prévisibilité, faible qualité juridictionnelle en matière commerciale) (p. 231). Les capitulations et leurs avatars ont donc servi de substituts à une réinterprétation ou une mise à jour du droit islamique (p. 250), avec pour conséquence une marginalisation croissante des marchands musulmans et des conséquences économiques et juridiques de long terme, dont la désislamisation du droit. En résumé, l'argument de $\mathrm{T}$. Kuran est que certains aspects de l'héritage institutionnel islamique (dispositions contractuelles, règles d'héritage, règles du mariage, rigidité du waqf) se sont progressivement transformés en faiblesses et ont incité ceux qui le pouvaient (particulièrement les bénéficiaires non musulmans du pluralisme juridique islamique) à opérer en dehors du champ du droit islamique. Aucune de ces institutions ne pouvait, à elle seule, faire du monde musulman un retardataire économique, mais leur imbrication et leur combinaison ont engagé celui-ci dans une dépendance trajectorielle rendant impossible la création d'institutions complémentaires, la capacité à l'innovation et à l'expérimentation, et une réaction efficace au sous-développement. La rhétorique du complot étranger et de la mécompréhension du droit islamique classique, qui prévalent souvent dans cette partie du monde, limite la marge de manœuvre des tenants de la réforme, de la globalisation et de la modernisation, et perpétue des débats stériles sur les vertus de l'islam comme solution à toute chose (p. 302).

L'argument de T. Kuran présente un grand intérêt. II nous montre en effet comment des institutions présentent des structures de possibilités, exploitées ou non, mais susceptibles d'exercer des effets de long terme. Ces mêmes institutions peuvent s'avérer dysfonctionnelles, toujours sur le long terme, sauf à les réformer, ce qui pose toujours un problème vis-à-vis des tenants d'une attitude conservatrice, renforcée par le caractère sacré des sources principielles. Il est bien évident ici que le "problème » ne vient pas de l'islam en tant que tel, mais de l'inscription de questions contingentes dans une structure de transcendance. Cette inscription freine les évolutions (pour le meilleur et pour le pire), qui restent toujours possibles mais entraînent un coût important et parfois exorbitant, sans parler des possibles retours au statu quo ante. La question demeure pourtant de savoir si, en utilisant la notion de «droit islamique », T. Kuran a facilité l'exposé de son argument ou l'a obscurci. Ce qu'il dit à propos du rôle de l'État, que l'historiographie a eu tendance à exagérer (p. 17), peut aisément être transposé au sujet du droit : la nature des archives, le caractère urbain de son développement dans des sociétés très majoritairement rurales, son caractère écrit dans un monde avant tout oral, autant de choses conduisant à exagérer sa surface, son impact, sa diffusion. Mais plus encore, l'idée d'un système juridique homogène et cohérent est conceptuellement problématique, parce 
qu'elle consiste à projeter, de manière anachronique et largement subreptice, sur un monde antérieur une conception propre au monde actuel. En somme, il eut été préférable de dire qu'il n'existait pas de droit commercial islamique plutôt que d'affirmer ses imperfections. Et c'est sans doute parce qu'un tel droit commercial islamique n'existait pas que, d'une part, l'irruption d'un droit commercial bien constitué a posé un tel défi et que, d'autre part, de nombreux juristes musulmans se sont échinés, depuis mais pas avant, à rassembler une série de dispositions attribuables plus ou moins aux sources de la doctrine islamique en un ensemble juridique homogène et intégré.

\section{Pluralisme et pragmatisme éclectique}

Dans son ouvrage Pragmatism in Islamic Law, Ahmed Fekry Ibrahim s'attache à explorer les usages et les évolutions d'une technique qui consiste à utiliser de manière éclectique le pluralisme juridique intrinsèque à l'islam - les quatre écoles de la doctrine sunnite - dans le but d'atteindre des objectifs pragmatiques (p. 3). II précise d'emblée que la notion de droit islamique renvoie à la fois au droit substantif (furu'), à la méthodologie juridique (usul al-fiqh) et à la doctrine (fiqh). Son « usage du [terme] "droit islamique" doit donc être compris comme faisant référence à l'éthique, au rituel et aux doctrines juridiques, ainsi qu'à la méthodologie et au lien qui les unit de sorte à appliquer la méthodologie juridique pour arriver à une compréhension (fiqh) du droit substantif » (p. 2). Sous-jacente à son exploration de cet éclectisme pragmatique, il y a une interrogation sur la charia et sur la trajectoire la menant de plain-pied dans la modernité, et donc une interrogation sur la réforme juridique en islam en tant que processus de création de lois modernes gardant intacte l'authenticité par le maintien d'un engagement vis-à-vis des sources scripturaires (Coran et Tradition). La technique de l'éclectisme pragmatique est utilisée aujourd'hui par des savants et des activistes qui font référence à l'ensemble de la tradition sunnite comme à un code unique dans lequel ils peuvent puiser pour répondre aux besoins changeants de la communauté musulmane. Certains auteurs, comme Wael Hallaq ou Nathan Brown, 1 considèrent que ce réformisme juridique contemporain, en contexte musulman, est inauthentique, au motif entre autre qu'il n'assurerait pas « la connexion organique du droit et de la société traditionnels » (p.6). Ils disqualifient l'éclectisme pragmatique par la même occasion, parce qu'il s'agirait d'un dérivé de la modernité. Pour W. Hallaq toujours, les règles traditionnelles que l'on retrouve dans les droits des États-nations ne sont que des vestiges inauthentiques, dès lors qu'ils sont déconnectés de la substance et de la méthodologie du fiqh classique (p. 219). Ahmed Fekry Ibrahim s'attache à montrer qu'il n'en est rien. Pour lui, s'il est sûr que la codification a créé une dynamique juridique profondément nouvelle et s'il est également sûr que l'on peut observer des transformations manifestes (disparition du waqf, nouvelle classe de juristes, passage d'un droit de jurisconsultes à un droit législatif), c'est néanmoins en termes de continuité et non de rupture qu'il faut réfléchir. L'éclectisme

\footnotetext{
1 W. Hallaq, The Impossible State: Islam, Politics, and Modernity's Moral Predicament, Columbia University Press, 2013; N. Brown, "Shari'a and State in the Modern Muslim Middle East", International Journal of Middle East Studies, 29:3, 1997, 359-376.
} 
pragmatique n'est qu'une nouvelle version des écarts classiques entre méthodologie juridique et droit positif, et seule une attitude nominaliste empêche de voir que la technique de l'éclectisme existait déjà dans la méthodologie classique, mais sous d'autres appellations (p. 8, 167, 177).

La technique de l'éclectisme pragmatique a été utilisée par les juges et les jurisconsultes comme un moyen de s'affranchir de la méthodologie juridique quand celleci ne permettait pas d'arriver au résultat désiré (p. 8, 135). Tout d'abord exclu, le pragmatisme juridique fut progressivement autorisé, ce qui renvoie à l'idée développée par l'historien du droit Alan Watson² qu'un droit peut procéder à des ajustements par voie de nouvelles divisions, exceptions, distinctions, dans le but d'étendre ou de restreindre le champ des lois existantes (p. 12-13). Pour Ahmed Fekry Ibrahim, c'est le modèle de la tradition vivante de la Common Law qui prévalait dans l'islam de la période initiale, jusqu'à ce qu'il soit remplacé par l'épistémè de la codification dans sa période classique. La fixation des règles propre à cette dernière (et donc l'inertie juridique qui en a résulté) a rendu nécessaire de ménager une ouverture au changement juridique, ce que fit l'éclectisme pragmatique (p. 15, p. 31-35). Cette technique, connue donc depuis longtemps, fut embrassée par certains et rejetée par d'autres. La question prit un tour plus aigu avec l'entrée dans la modernité.

Au début du XX $x^{e}$ siècle, Rashid Rida identifiait, s'agissant de la fabrique du droit, cinq groupes différents, qu'Ahmed Fekry Ibrahim reprend à son compte en en changeant la dénomination : les traditionnalistes, qui rejettent l'interprétation réformatrice puisant à l'extérieur des sources doctrinales d'une école mais acceptent l'éclectisme interne à celle-ci ; les sécularistes, qui n'acceptent pas l'islam comme source de droit ; les réformateurs, qui prétendent accommoder l'islam et la modernité en cherchant une « voie moyenne » entre les deux extrêmes ; les puristes, qui rejettent les écoles, toute forme d'incrémentation humaine et l'inspiration étrangère des discours modernes ; les libéraux, qui prétendent développer de nouveaux paradigmes herméneutiques compatibles avec les principes démocratiques, dans le but de moderniser l'islam (p. 182-183). Les puristes, généralement qualifiés de «salafistes 》 aujourd'hui, sont prompts à mettre l'accent sur la charia dans son état originel, préservée de l'interprétation humaine, qui ne peut être découverte que par le biais d'une interprétation nouvelle des textes sources et par l'évaluation des règles correctes à l'intérieur des opinions déjà existantes, en évaluant les preuves disponibles, en excluant les opinions contraires aux traditions (hadith) et en répudiant les écoles doctrinales. Ces puristes rejettent toute référence à la modernité, qui serait une forme de sécularisation que le processus de codification a déjà entamée (comme dans l'usage de l'éclectisme pragmatique pour codifier le statut personnel) (p. 189-190). Dans cette perspective, le droit islamique est tenu pour strictement équivalent au sens apparent des textes sources tels qu'interprétés par les savants autorisés. Pour les tenants de cette ligne, les réformistes ne sont que des sécularistes se cachant derrière un islam de façade. Ces réformistes, qu'il s'agisse de Qaradawi ou de Ghannushi, ont un rapport sélectif et créatif par rapport aux textes sources dont ils cherchent les

2 A. Watson, Legal Transplants: An Approach to Comparative Law, University of Georgia Press, 1993. 
correspondances avec les principes de la démocratie libérale (p. 195). Quant aux libéraux, ils entreprennent de redéfinir l'herméneutique et les textes sources, généralement en s'affranchissant de la littérature des hadiths ou en se centrant sur la substance des traditions qu'ils mettent en perspective de l'intention coranique (p. 198).

L'éclectisme pragmatique s'est davantage imposé dans le discours moderniste que libéral, les tenants du modernisme ayant réussi à tenir l'argument que leur approche s'appuyait sur l'autorité de la tradition juridique prémoderne (p. 200). La codification partielle du droit de la famille inspiré de la charia, en Égypte, correspond, pour Ahmed Fekry Ibrahim, à un retour au pluralisme juridique pragmatique antérieur au XIX siècle. La loi de 2000 sur le khul' est un révélateur de cette ambivalence : si, d'un côté, il s'agit d'une « discontinuité radicale » par rapport au droit islamique (Arabi), voire un exemple manifeste d'occidentalisation (Muhanna), ${ }^{3}$ il n'en demeure pas moins, de l'autre côté, qu'elle atteste de la préférence du législateur pour l'adoption de textes dérivant leur autorité de la doctrine d'une école établie et que, même sous le gouvernement des Frères musulmans, elle n'a pas été abolie (p. 211-212). De manière intéressante, l'auteur montre que le glissement qui s'est opéré, dans le processus de modernisation juridique, tient à l'accent mis sur le contenu des règles plutôt que sur leur méthodologie. Ce qu'on appelle aujourd'hui le « retour à la charia », c'est une remise en vigueur du droit islamique substantiel (p. 222), ce qui implique par la même occasion que la charia, telle que comprise par la majorité de ses tenants, est déjà très largement « restaurée ». Tout en reconnaissant qu'après sa codification, le droit islamique ne sera plus jamais le même (p. 229), Ahmed Fekry Ibrahim conclut que, pour l'essentiel, le droit actuel - dans le domaine de la famille à tout le moins - consiste, pour les législateurs, à puiser de manière éclectique dans les écoles du droit islamique classique afin de satisfaire les exigences du monde moderne (p. 235).

Le travail d'Ahmed Fekry Ibrahim est, en plusieurs points, remarquable : documentation, maîtrise de l'histoire doctrinale et judiciaire en contexte arabo-musulman, mise en relation des périodes classique et moderne. II n'en pose pas moins de gros problèmes qui sont, comme dans le cas de Timur Kuran (cf. supra), avant tout conceptuels. Sa conception extensive à l'extrême du terme “droit » le conduit à des comparaisons d'autant plus hasardeuses qu'elles confondent le travail des jurisconsultes et celui des juges. En soulignant à plusieurs occasions le bouleversement induit par la codification et en parlant de passage de droit processuel à droit substantiel, Ahmed Fekry Ibrahim avait l'occasion de montrer à quel point il conviendrait davantage de parler de révolution juridique. II ne le fait toutefois pas, parce que cela irait contre son argument de continuité. Mais son argumentation se fait à front renversé : ce n'est pas le droit islamique d'aujourd'hui qui plonge ses racines dans le fiqh d'autrefois, mais bien le fiqh d'autrefois qui trouve des prolongements dans le droit substantiel d'aujourd'hui. Autrement dit, si continuité il y a, ce n'est pas dans le concept de droit, mais dans la substance de certaines règles, doctrinales hier, juri-

3 O. Arabi, "The Dawning of the Third Millennium on Shari'a: Egypt's Law No. 1 of 2000, or Women May Divorce at Will", Arab Law Quarterly, 16(1), 2-21; M. Muhanna, "Qanûn al-Ahwâl al-Shakhsiyya", Al-Muslim Magazine, Mars 2000. 
diques aujourd'hui. La question n'est évidemment pas, comme le font accroire W. Hallaq ou N. Brown, celle de l'authenticité - l'authenticité n'est pas une propriété essentielle des choses mais un attribut qu'on leur confère - mais celle d'une révolution de la normativité. Les puristes ne s'y d'ailleurs pas trompés, eux qui voient dans la codification un fait de sécularisation. Quand Ahmed Fekry lbrahim procède à une comparaison qu'il veut heuristique entre le droit islamique primitif et la Common Law, tout d'abord, et entre le droit islamique classique et le droit civil, ensuite, il commet une erreur de catégorie qui produit de la confusion conceptuelle. Cette comparaison induit en effet le cadre d'analyse et, plutôt que de clarifier la question, la brouille en contraignant les termes à entrer dans un schème qui ne leur correspond pas. En outre, l'auteur tend à se centrer sur des exemples marginaux au regard de l'évolution du droit de la famille égyptien : des auteurs de doctrine, alors que le pouvoir législatif est passé en d'autres mains ; des cercles religieux, alors que la loi se décide dans les plus hautes sphères de l'État. En somme, ce n'est pas le droit islamique qui a changé de nature, mais le fiqh islamique qui s'est transformé en droit.

\section{Continuité et rupture, à moins que ce ne soit l'inverse}

Faire l'histoire du droit colonial, dirigé par Jean-Philippe Bras, nous conduit à l'exploration d'un autre cas de figure, celui d'un droit inscrit sur une période précise, le temps colonial, et donc dans une discontinuité flagrante entre l'avant précolonial et l'après des indépendances, qui n'est pas pour autant sans faits et effets de continuité : pérennité des «droits » musulman et coutumier, d'une part, et perpétuation du modèle civiliste, de l'autre. Recueil de contributions de qualité variée, l'ouvrage vaut tout particulièrement par son introduction et par les chapitres consacrés au droit foncier, aux magistrats et aux avocats, à l'expérience particulière d'une revue, aux premiers temps de l'indépendance algérienne et au droit colonial algérien lui-même. Si l'on commence par le moment colonial, on remarquera, avec Claude Bontems, que le «droit musulman » algérien est un droit inventé, souvent par des tandems faits d'islamologues et de juristes, les seconds donnant aux premiers le cadre cognitif d'appréhension du matériau brut que les premiers sont appelés à traduire dans le langage du droit (p. 309). De ce point de vue, le droit colonial a bouleversé le cadre normatif indigène, sous prétexte de systématisation du droit antérieur, alors qu'il s'agissait en fait de construction d'un droit discriminatoire à frais nouveaux. L'exemple du droit foncier est parlant. Jean-Philippe Bras en démonte remarquablement les mécanismes. Loin de permettre une lecture univoque de la domination coloniale, il en illustre bien davantage les ambiguïtés et ambivalences (p. 86). Avant toute chose, il faut remarquer que la colonisation française repose sur des principes intenables : la création d'un empire à partir d'un État-nation ; une disparité de droits contestée par un ethos universaliste (p. 87). Voici donc les juristes tenus de fonder en droit un régime de propriété incompatible avec l'économie du projet colonial, avec en conséquence l'instauration d'un système dual, entre régime foncier certifié à la française et régime foncier « musulman » incertain ouvert à la captation. Ce dernier s'explique, dans le langage colonial, par les traits culturels propres à une civilisation inachevée - et donc colonisable et civilisable (p. 89). Les opérations 
d'immatriculation et de francisation sont très symptomatiques de cette ambivalence, elles qui font accéder les indigènes à des droits de propriété modernes, mais en même temps les dépossèdent de leurs instruments et juridictions propres (p. 96). La doctrine opère, dans ce contexte, par une forme de balancement qui renvoie l'indigène à ses formes traditionnelles et en même temps lui permet d'accéder à une modernité conditionnelle (p. 109), ce que souligne également Jean-Robert Henry, pour qui les textes juridiques coloniaux rusaient avec les principes égalitaires du droit français pour les accommoder aux situations de domination coloniale (p. 226). L'on voit ainsi que la prétention universaliste du droit se trouve en permanence contrée par la nécessité de conserver la catégorie d'indigène, sans laquelle la domination coloniale ne se peut plus. Et la notion de droit musulman, dans tout cela, permet de perpétuer la catégorie d'indigène et parfois même d'agir, à front renversé, dans un sens contraire à ses intérêts (en matière foncière, p. 100) ou carrément régressif (en matière familiale, p. 192). Comme le montre Florence Renucci, ce mécanisme de saisie de la norme islamique dans les termes du droit français a conduit un juriste comme Morand à formuler la théorie de l'immutabilité du droit musulman et à, par exemple, traduire des ouvrages du XIV siècle devant servir de référence dans l'administration de la justice, avec la conséquence paradoxale d'un droit colonial « réislamisant » le droit musulman (p. 192). La Revue algérienne fut à cet égard très instrumentale, tout en étant aussi l'écho régulier du dilemme des juristes confrontés à un intérêt colonial préféré à la logique juridique (p. 195). Parmi ces juristes, une mention toute particulière doit être faite du magistrat qui, comme le fait remarquer Nada Auzary, est l'acteur principal d'un régime colonial dont le droit est l'instrument (p. 139). Le magistrat colonial exerce aussi une fonction législatrice : la décision qu'il rend s'appuie sur une norme qu'il a contribué à créer, entre autre par le commentaire qu'il en fait (p. 147). II s'inscrit dans un processus d'acculturation, à côté de l'enracinement de la culture de l'écrit, de l'apparat judiciaire et de l'architecture des bâtiments de la justice (p. 155). Enfin, sa subordination au pouvoir exécutif, sa précarité et sa mission civilisatrice en font l'instrument précaire d'un pouvoir avant tout bureaucratique.

Si l'on examine, en aval du temps colonial, celui des indépendances, on remarque, à la suite de Jean-Claude Vatin, que la rupture politique ne s'accompagne pas vraiment d'une rupture juridique, dans le cas algérien à tout le moins. Certes, l'appareillage juridique est à adapter dans l'urgence, mais cela se fait à partir du droit colonial et du droit français, et avec un personnel formé à ces droits et en français (p. 54). En outre, certaines des caractéristiques de la culture politique et juridique de l'ancien colonisateur s'avèrent attractives, comme le centralisme étatique, le républicanisme intransigeant et la monopolisation de la contrainte (p. 55). Même constat pour Jean-Robert Henry, qui souligne que, par crainte du chaos et du vide juridique, les nouveaux responsables algériens ont veillé à ne pas détruire le système normatif existant, dont l'existence se révélait d'ailleurs un outil précieux pour la transition vers un nouvel ordre ( $p .225)$. Cette reproduction de modèles hérités de la période coloniale se retrouve aussi dans la régulation et le fonctionnement des professions judiciaires, comme le montre Éric Gobe à propos du barreau tuni- 
sien (p. 162), quitte à ce que des lois donnent aux instances ordinales les instruments de contestation d'un État aux velléités autoritaires (p. 178). L'enseignement du droit s'adapte également, plutôt qu'il ne rompt avec le passé. Ainsi, le cours de "législation algérienne » est-il remplacé par celui d' " histoire des institutions algériennes » (p. 234). Quant au droit musulman, dans ce processus, il n'est plus qu'un chapitre du droit civil, dès lors qu'il avait été intégré au droit positif (p. 236). Il convient donc d'être prudent en parlant de rupture avec le passé colonial et avec son droit, qui, bien que devenu sans objet, n'en trouve pas moins des prolongements sous différentes formes. Est-ce à dire qu'il faille embrasser le paradigme postcolonial, qui met l'accent sur la reproduction des marqueurs de domination et, sur cette base, instruit le procès du droit colonial et de son héritier contemporain ? Dans son introduction, JeanPhilippe Bras montre à la perfection qu'il y a là une caricature dont il faut se garder, parce qu'elle est généralisatrice et réductrice à l'excès de l'objet colonial ( $p .13)$. Ce travers s'explique par l'excès foucaldien de la méthode qui sort l'objet colonial de son contexte historique en en postulant l'unicité et l'invariabilité (p. 14). À cette unicité postulée du droit colonial, on peut opposer la diversité de ses formes, le mélange de ses composantes (métropolitaines, spécifiques et locales) et les incertitudes quant à son statut. À son exceptionnalité, on peut opposer l'usage des mêmes registres qu'en métropole. À son unilatéralité (un droit des dominants), on peut opposer le caractère pluriel et empilé de sa structure. À la perpétuation des schèmes de domination que les droits colonial et postcolonial assurent, on peut opposer leur nature infiniment plus composite (p. 16-21). II s'agit en somme de faire remarquer le tournant crucial mais nuancé et complexe que constitue le droit colonial dans la vie normative des sociétés colonisées (et colonisatrices), dans la rupture qu'il constitue lors de sa mise en place et dans ses modes de perpétuation ensuite.

L'ouvrage sur le droit colonial intervient à point nommé dans notre discussion, dans la mesure où la rupture dont il traite est explicite, là où différents effets, dont ceux du nominalisme, conduisaient les livres sur le droit islamique à davantage insister sur l'idée de continuité historique et sémantique. On y retrouve très clairement l'idée qu'en amont, le droit colonial, même dans les domaines où il se pose dans une continuité, comme en matière personnelle ou foncière (surtout sur la question des terres collectives), consiste en l'imposition d'un nouveau cadre cognitif dans l'intelligence d'une matière normative certes labourée depuis longtemps, mais selon une grammaire toute différente. On y retrouve aussi l'idée qu'en aval, l'héritage du droit colonial n'a pas opéré sur le mode de la rupture, mais a plutôt accompagné dans la continuité le changement, lui radical, de régime politique. À différents égards, droit islamique et droit colonial ont partie liée, tant il est vrai que le traitement de la normativité islamique en termes de droit est intimement lié à ce $\mathrm{XIX}^{\mathrm{e}}$ siècle que l'on a pu qualifier de siècle du droit. Et si le droit colonial s'avère être un complexe empilement de législations aux sources et objectifs disparates, c'est sans doute parce que, précisément, il consiste en la volonté d'appliquer un concept - présenté comme universaliste mais en réalité situé et connoté historiquement - à une réalité qui n'est pas celle de son terreau de gestation. 


\section{Le juridisme}

Une série de séminaires sur le thème du « legalism », que nous rendons par « juridisme ", sont à l'origine de la production de trois ouvrages d'anthropologie historique et comparative d'une qualité sans précédent. Nous nous intéresserons plus particulièrement à deux d'entre eux. En introduction au premier d'entre eux, Legalism: Anthropology and History, Paul Dresch, véritable tête pensante de l'entreprise, définit cette notion comme « l'ensemble des thèmes impliquant un appel à des règles distinctes de la pratique, un usage explicite de concepts généralisants et une disposition à traiter dans ces termes la conduite humaine » (p. 1). II remarque que cette notion ne coïncide pas nécessairement avec celle de système juridique développée par Joseph Raz. Son ambition est de saisir, sous l'appellation de juridisme, ce que le droit signifie, pour qui et dans quel contexte (p. 8). La notion de juridisme semble permettre d'échapper à certaines apories de la définition du droit en se penchant sur l'air de famille qu'une façon de raisonner partage universellement. Comme le fait remarquer J. Raz, " les règles propres aux associations privées et celles propres à l'État moderne fonctionnent de manière similaire, mais seules les secondes constituent du «droit » en ce qu'elles sont englobantes et suprêmes » (cité par Paul Dresch, p. 15). II n'est toutefois pas sûr que P. Dresch ait pleinement apprécié ce que permettait et empêchait sémantiquement son choix terminologique et, en tout cas, il n'en a pas tiré tout le parti possible, bien que nombre de ses remarques s'avèrent d'une pertinence toute particulière. Ainsi en est-il de sa critique des théories du pluralisme juridique qui assimilent le droit au contrôle social (p. 9) ou feraient du droit un miroir de la société, alors qu'il s'agit plutôt d'une carte permettant de vivre en société sur la base de règles catégorielles (p. 161). Ainsi en va-t-il aussi de son insistance sur le fonctionnement catégoriel du juridisme : les catégories juridiques fournissent les costumes et les rôles dans lesquels inscrire les personnes, les actions et les événements (p. 13). Le juridisme, dans cette perspective, c'est " le traitement du monde à travers des catégories et des règles, explicites ou non, qui sont distinctes de la pratique » (p. 15), des «concepts catégorisants " pour reprendre les termes d'Edward Levi (cité par Paul Dresch, p. 25). Le raisonnement juridique spécialisé, dont il situe l'apparition au tournant du Moyen Âge tardif, consiste à relier des concepts juridiques les uns aux autres dans une jurisprudence reconnaissable (p. 17). On observe, à cette époque, la volonté de quadriller, en les définissant, l'ensemble des relations humaines dont on souhaite exploiter toutes les implications logiques (Strayer, cité par Paul Dresch, p. 17). C'est à ce moment que le droit s'autonomise du sens commun (p. 19-20) et devient une « théologie de la vie quotidienne » (Davis, cité par Paul Dresch, p. 21), c'est-à-dire une rationalisation de l'expérience et de ses formes. Le juridisme, en ce sens, est un ordre conceptuel (p. 33), où les mots sont performatifs en ce sens qu'ils créent des catégories nouvelles (voir Judith Scheele, p. 227). Dès lors, comme l'indique P. Dresch, ce que toutes les formes de juridisme ont en commun, c'est de « cartographier et organiser le monde en termes qui ne se réduisent pas à la rencontre de personnes spécifiques. Les catégories et les règles invoquées sont, à l'inverse, supposées être applicables à toutes les instances de même type, ce qui suggère une conception de la vie, même locale, plutôt différente de la simple familiarité 
avec ses parents et ses voisins » (p. 25). Du fait de sa nature catégorisante, le droit fonde un système d'appartenance et d'exclusion des personnes (p. 33), de même qu'un principe de division du temps et de l'espace (p. 34). Paul Dresch poursuit en soulignant la différence qu'il existe entre les règles particulières (laws, Gesetze) et le droit (law, Recht) (p. 155). Le droit, nous dit-il, est marqué par l'idéologie moderne qui fait de sa conception le standard par rapport auquel les autres expériences doivent être évaluées (p. 30).

De nombreuses autres contributions à l'ouvrage s'avèrent d'un intérêt particulier. Ainsi en va-t-il de la définition du juridisme que nous donne Hannah Skoda: des règles distinctes de la pratique; des règles qui sont plus que l'improvisation; des règles qui sont systématiques (p. 39). Reconnaissant que le terme de «droit » est polysémique et fluide (p. 40), H. Skoda insiste sur le fait que des différences terminologiques peuvent celer des distinctions opératoires (renvoyant à Georgy Kantor, p. 69-70, qui montre par exemple qu'il n'existe pas de terme générique, dans les traditions hellénistique et romaine, pour parler des droits personnels, qu'il n'existe pas de terminologie juridique à proprement parler dans la tradition hellénistique, et que la terminologie juridique romaine était plus formelle que basée sur un contenu ; voir aussi Paul Dresch, p. 147, qui insiste sur la nécessité de contextualiser l'usage des mots). Et il faut bien reconnaître que parler de droit sans le définir ne peut mener et n'a mené qu'à la confusion (Fredric Cheyette, cité par Georgy Kantor, p. 66). La question se pose alors de savoir s'il faut définir conceptuellement le droit a priori ou s'il convient plutôt d'en recourir aux termes avec lesquels la normativité se trouve catégorisée dans chaque cas d'étude et de chercher les traductions et correspondances qui lui correspondent le mieux. Montesquieu (cité par Malcolm Vale, p. 278) ne disait-il pas déjà qu'il n'existe pas de principe supérieur ou de principes particuliers pouvant expliquer, et encore moins déterminer, les manifestations variées du droit et de la coutume?

Les utilitaristes et les positivistes ont réussi à imposer une conception du droit comme un code rationnel de loi créé et appliqué par l'État, et coupé de toute visée éthique nécessaire (voir Donald Davis, p. 88). Le même D. Davis montre que le langage des droits émane des théories politiques de Locke et Hobbes, et culmine dans les révolutions américaine et française ; et aussi que nous sommes aujourd'hui tellement habitués au processus judiciaire fixé par l'État que nous en avons oublié la possibilité de parler autrement du droit (p. 87). Pour différents auteurs du livre, pourtant, ce même juridisme répond, selon les latitudes, à des visées variées : discours sur la rectitude morale et religieuse en Inde médiévale (Donald Davis, p. 86,) ; rôle " aspirationnel » dérivé de sa capacité à inscrire la vie locale dans le cadre universel d'une civilisation et d'un ordre vertueux dans le cas du Touat algérien (voir Judith Scheele, p. 198, pour qui la charia constitue une opportunité d'accès à un champ d'action politique et économique plus vaste, de même que d'appartenance à un monde plus large fondé sur des prétentions à la vérité universelle et à la permanence) ; idée d'un répertoire rhétorique et argumentatif définissant, en-dehors même des instances judiciaires, le juste et le faux (voir l'introduction de Hannah Skoda, p. 43, renvoyant à son chapitre dans le même volume). La notion de juridisme 
semble donc heuristique, en ce qu'elle permet de clarifier certaines associations conceptuelles problématiques. II en va de la sorte des liens unissant les règles et catégories formant l'appareillage conceptuel du juridisme, d'une part, et la recherche des faits dont dépend une bonne part de l'application du droit, de l'autre (introduction de Hannah Skoda, p. 43). II en va de même de l'association du juridisme et de l'écriture, la question de la compréhension du contenu des textes juridiques étant parfois moins importante que leur préservation comme des artéfacts incarnant un statut et une appartenance (renvoyant à Judith Scheele, même volume, et aussi à son propre chapitre, p. 300, où il apparaît que la systématisation du droit s'adosse à l'idée que celui-ci est quelque chose de raisonnable de manière inhérente, chose qui se trouve renforcée par la stabilité que permet l'écriture). II en va ainsi aussi de l'association du juridisme et de l'importance croissante de l'État ou d'un pouvoir de coercition centralisé (voir Hannah Skoda, p. 41 et 52). Un auteur comme Patrick Wormald (cité par Donald Davis, p. 87) considère, à cet égard, qu'il est erroné de parler de «systèmes juridiques » et qu'il faudrait remplacer cette notion par celle de “cultures juridiques ». Un autre auteur comme Simon Roberts (cité par Paul Dresch, p. 151) souligne, pour sa part, que le processus de centralisation cristallise les compréhensions, pratiques et formes institutionnelles que nous en sommes venus à associer au concept de droit, lequel, dans une tradition toute kantienne, dérive en entier d'une autorité centrale. Aujourd'hui, l'anthropologie contemporaine ne conçoit le droit qu'en relation à un pouvoir qui oblige à son obéissance (Paul Dresch, p. 166). II en va également ainsi du lien unissant droit, moralité et pouvoir, un véritable nœud qu'il convient de desserrer (p. 145).

\section{Règles et catégories}

Legalism: Rules and Categories, l'autre volume sur le juridisme, porte plus spécifiquement sur la relation que cette notion entretient avec les concepts de catégories et de règles, les deux étant indissociablement liés, au point de dire qu'en définissant le champ d'application d'une catégorie, on joue directement avec et sur des règles (voir Paul Dresch, p. 67). Au-delà du juridisme, l'introduction insiste sur le fait que c'est l'objectif même des sciences sociales que d'identifier et de conceptualiser les catégories qui sous-tendent la pensée et l'action (p. 5). En arrière-plan de l'ouvrage, on peut remarquer le recours, inhabituel en anthropologie et en histoire, aux débats de philosophie et théorie du droit. II est ainsi fait référence à Lawrence Friedman, pour qui le juridisme se définit comme le raisonnement menant à concevoir la vie en termes de règles et à suivre des règles (p. 1). Ce qui ferait le propre du raisonnement juridique, nous dit-on, c'est précisément l'usage de catégories explicites inspirées du sens commun à l'intérieur d'un système conceptuel distinct ( $p .11)$. Les règles seraient donc des concepts généralisants distincts de la pratique (p. 7). C'est, à notre sens, une erreur de vouloir trop détacher règles et pratiques, alors que la question à étudier ne relève pas de la dichotomie mais de la relation : pas de règle sans pratique de la règle, pas de pratique d'une règle sans énoncé/énonciabilité de celle-ci. Sans doute Paul Dresch commet-il aussi cette autre erreur de confondre règles en général (qu'il définit comme des principes ayant une force normative, p. 66) 
et règles de droit (dont il voit l'apparition comme le résultat d'un processus évolutif, p. 59). On peut avoir des réserves à l'égard de ce formalisme, que ne confirme d'ailleurs pas la lecture des travaux de Frederick Schauer sur lesquels Paul Dresch s'appuie beaucoup. La règle ne peut être détachée de la pratique de la règle. La référence à la règle, même quand on l'enfreint, et la survenance au moins exceptionnelle d'un cas d'application de celle-ci semblent ainsi constituer les conditions minimales de son existence. Certes, dans un système lié par des règles, celles-ci précèdent les événements auxquels elles se rapportent (bien que leur édiction puisse être le fruit d'une déduction à partir d'événements passés), mais ces événements sont précisément des cas d'application de la règle et définissent donc en retour les règles mêmes dont ils sont les applications.

L'anthropologie est convoquée, dans cette présentation, pour l'exploration des supposés culturels fondant les rapports locaux au règles (voir l'introduction, p. 12), mais aussi pour souligner que la soumission aux règles relève souvent plus de l'acceptation d'un système sociopolitique dans son ensemble et non dans le détail (p. 22). La discipline anthropologique contemporaine est plutôt réticente à l'idée de règle. Pourtant, s'il est évident que nombre de nos activités ne sont pas contraintes par des règles, il ne faut pas y voir une justification pour ignorer leur incidence dans la vie en société. De ces règles, il est possible de faire la grammaire, logique et pratique, et cela à son tour reflète la façon dont l'action est, en contexte et in situ, conçue et catégorisée (p. 25). Mais que recouvre donc le concept de règle ? Paul Dresch en observe le «champ sémantique sauvagement hétéroclite » (p. 53), mais prétend également, on l'a dit, à leur existence autonome de la pratique. Est-ce à dire qu'une règle systématiquement enfreinte reste une règle, comme il l'affirme plus loin (p.56) ? Notons toutefois que Paul Dresch est également soucieux d'affirmer le caractère contingent des règles, dont celles de droit, à l'encontre de l'historicisme de nombreux juristes (p. 67). Caroline Humfress va dans le même sens dans sa critique du pandectisme et de son idéalisme déductivo-rationaliste, qui prétendait identifier les principes de bases de tout système juridique (p. 81). Les deux auteurs convergent, de ce fait, sur la nécessité de ne pas opposer stérilement formalisme et sociologisme, et d'engager tout à l'inverse une ethnographie du raisonnement juridique (p. 89), à l'instar de ce que préconisait Jhering au XIXe siècle déjà (p. 85). C. Humfress affirme en conséquence que concepts juridiques et descriptions sociologiques vont de pair, les constructions conceptuelles devant être étudiées dans le contexte des sociétés les ayant produites (p. 87) : les concepts et les règles ne flottent pas en apesanteur, en-dehors de leurs propres pratiques, pas plus qu'ils ne fonctionnent de manière purement instrumentale (p. 103). À cet égard, Judith Scheele insiste à juste titre sur le fait qu'il n'est possible pour l'anthropologie de parler de règles qu'à la condition que l'observateur et l'observé jouent au même jeu, ce qui signifie concrètement, sur le thème qui nous occupe, que le compte rendu ethnographique d'une société par le biais des règles qu'elle observe suppose que, pour cette société précise, le jeu des règles soit à tout le moins imaginable (p. 165). Cette même observation pourrait être étendue au droit : pourquoi parler de droit à propos d'une société qui ne se conçoit pas dans ces termes? 
S'il est vrai que la théorie juridique assimile trop facilement le droit aux règles (voir l'introduction, p. 21), il faut remarquer en même temps que l'ouvrage entier fait du juridisme l'incarnation du raisonnement « axiologique » (par les règles). Ainsi Donald Davis nous dit-il que, dans la tradition sanskrite, les règles expriment la ligne de partage entre le monde social et le monde non social (p. 29). Caroline Humfress entend, pour sa part, le « juridisme » comme «soumission aux règles » (rulefollowing, p. 79). Et Judith Scheele définit le juridisme comme une façon de se saisir du monde dans des termes généralisants, ce qu'elle qualifie de « suppositions locales sur la moralité » (p. 153). On ne peut manquer de souligner à quel point l'assimilation des règles au seul droit est inadéquate. Sans doute serait-il d'ailleurs préférable de parler d' « axiologisme » plutôt que de « juridisme ». Le choix du vocabulaire est donc crucial. Fernanda Pirie, par exemple, utilise le terme de juridisme pour rendre compte d'un discours formulé en termes de règles et de catégories explicites (p. 106) et Morgan Clarke décrit le discours de la charia au Liban comme un prototype de juridisme (p. 232). II s'agit d'une erreur de catégorie, ou plus exactement de niveau taxinomique, qui aurait pu être évitée en ne s'attachant pas à parler du droit et du juridisme, mais de la norme ou de la règle et du formalisme, comme le fait d'ailleurs remarquer Elizabeth Ewart, quand elle dit que « l'explication de ce qui est requiert de se tenir à l'écart d'une idée telle que celle de droit » (p. 208). Nous parlons de niveau taxinomique pour insister sur le fait que la catégorie « règles de droit » appartient à la catégorie supérieure « règles », et non l'inverse, et que les deux catégories ne sont donc pas de même degré. S'il est parfaitement exact, de ce point de vue, de parler du discours de la charia comme d'un archétype d' " axiologisme » (centralité de règles et catégories explicites), il est faux, à moins de pousser dans des aboutissements absurdes l'analogie entre lois des hommes et Loi de Dieu, d'y voir l'incarnation du juridisme (une tradition intensément « juridiste »; voir Morgan Clarke, p. 256).

\section{Le concept de droit n'est pas universel}

Les deux ouvrages dirigés par Paul Dresch, l'un avec Hannah Skoda et l'autre avec Judith Scheele, sont à tous égards passionnants et soulèvent un grand nombre de questions d'importance, faute de toujours y répondre adéquatement. On l'a dit, la notion même de "juridisme » aurait pu être mieux exploitée, particulièrement sa capacité à déjouer les apories propres à tout exercice de définition analytique du droit. En plusieurs endroits, les choses sont dites, mais on n'en tire pas pour autant les conséquences. Ainsi en va-t-il quand on insiste sur l'impossibilité d'échapper aux représentations contemporaines et occidentales centrées sur l'État et le droit positif (voir Donald Davis ${ }^{4}$, p. 87). Ainsi en va-t-il aussi quand on en appelle à une définition perspectiviste et contextuelle du droit (Georgy Kantor, vol. 1, p. 66). Parler de juridisme plutôt que de droit, c'est aller dans la bonne direction, en ce sens que cela renvoie à une forme de pensée ou de raisonnement plutôt qu'à un système normatif

4. In Paul DrESCH et Hannah SKodA (eds.), Legalism: Anthropology and History, op. cit., ci-après désigné par « vol. $1 »$. 
spécifique. En même temps, l'usage d'un terme dont l'étymologie renvoie à celui de droit crée à son tour un halo de confusion. En fait, on assiste le plus souvent à une inversion conceptuelle: alors qu'il faudrait parler du droit comme d'une instance particulière de la catégorie générique de la normativité, on en fait la catégorie générique recouvrant toutes les instances particulières de normativité. II en est alors du droit comme de ces autres termes du langage ordinaire dont la science s'empare pour les sophistiquer et les transformer en catégories analytiques. Pourtant, on pourrait tout aussi bien remarquer l'existence de jeux de langage partageant un air de famille, celui de la normativité, sans que cela ne justifie de faire accéder le terme de droit à un statut analytique qu'il n'a pas (ou seulement alors au prix de distorsions confondantes). Avancer dans l'étude du droit, c'est peut-être tout d'abord consentir à faire un pas en arrière et entreprendre un travail à la fois analytique et ethnographique d'étude de la normativité et de ses éléments constitutifs principaux, les règles. À cet égard, les enseignements de l' «Analytical Jurisprudence » restent pleinement d'actualité, surtout quand elle affirme que concepts juridiques et description sociologique vont de pair, les constructions conceptuelles devant être étudiées dans le contexte des sociétés les ayant produites (voir Caroline Humfress 5 , p. 87) : les concepts et les règles ne flottent pas en apesanteur, en-dehors des pratiques, pas plus qu'ils ne fonctionnent de manière purement instrumentale (ibid., p. 103). Ce simple pas en arrière - si l'on est d'accord sur le fait que les règles ou les normes sont, comme l'affirme Herbert Hart, les blocs de construction du droit - a pour effet de dissiper le brouillard conceptuel créé par l'usage d'un terme contingent et chargé historiquement. II ne subsiste alors plus aucun problème à parler, par exemple, de la nature «aspirationnelle » (voir Judith Scheele, vol. 1 ; Fernanda Pirie, vol. 2, p. 105) de certaines normes et règles, dès lors que, délestées de leur prédicat de «juridiques ", elles retrouvent leurs caractères et finalités propres qui, d'un contexte à l'autre, peuvent être spécifiques ou communs, divergents ou convergents, universels ou locaux, régulateurs ou idéalistes, contraignants ou indicatifs, etc. Si l'on ne le prend que pour un cas d'application contingent d'un jeu de règles entendues sensu lato, il est beaucoup plus facile aussi de parler du droit stricto sensu. À l'inverse, dès lors que l'ombre du droit ne plane plus sur leur usage et leur examen, les concepts de norme et de règle peuvent à nouveau être abordés librement, comme le fait Winch, par exemple, pour qui tout comportement doté de sens est ipso facto articulé autour de règles, que celles-ci soient explicites ou implicites (cité par Judith Scheele, vol. 2, p. 169). Parler de règles et de normes plutôt que de droit autorise une marge de manœuvre analytique embrassant un éventail de phénomènes bien plus large que le seul phénomène juridique, et dans des termes moins ambigus également. Cela permet, par exemple, de traiter des catégories du système médiéval de relations sociales en dehors du carcan juridique qui ne leur convient pas (voir Alice Rio, vol. 2, p. 150) et d'ainsi mieux rendre compte de l'usage de termes intermédiaires offrant, à l'époque, une plus grande plasticité et donc marge d'interprétation, loin de

5. In Paul DreSCH et Judith ScheELE (eds.), Legalism: Rules and Categories, op. cit., ci-après désigné par « vol. $2 »$. 
l'ordonnancement logique que suggère le concept de droit (Fernanda Pirie, vol. 2, p. 121).

Si l'on s'attache à vouloir définir ce qu'est le droit, universellement et analytiquement, on commet le plus souvent un anachronisme qui consiste à exhumer du passé les éléments qui correspondent à notre expérience d'aujourd'hui. Si nous voulions trouver une définition analytique et universelle, il nous faudrait procéder à l'inverse, chose naturellement impossible : nous pouvons parler du droit babylonien à partir de nos catégories d'aujourd'hui et donc de notre concept contemporain de droit, mais nous ne pouvons déduire notre concept de droit contemporain de l'ensemble des expériences historiques de la normativité qui ne se pensaient pas dans les termes du droit d'aujourd'hui. Autrement dit, un concept tel que celui de droit ne peut être détaché de l'actualité à laquelle il fait référence. De ce point de vue, le concept de droit n'est ni analytique, ni universel, mais historique et contingent. Et c'est parce qu'il est tel qu'on peut en parler comme, par exemple, doué d'un idéal de complétude et de cohérence (Fernanda Pirie, vol. 2, p. 119), ou qu'on peut dire, à l'inverse, que « parler du "droit" en Russie ancienne, en utilisant l'article défini (the) de l'anglais, nous égare » (Simon Franklin, vol. 2, p. 196). II n'en va pas de même d'un concept tel que celui de morale, entendu comme démarcation du bien et du mal. Celui-ci est également normatif et requiert une capacité de jugement de ce qu'est la bonne chose à faire, mais, à de rares exceptions près 6 , se retrouve dans tous les types de configurations sociales possibles et imaginables.

Le droit est bien le produit de l'idéologie moderne, occidentale et ethnocentrique, centralisée, positiviste, génératrice d'une conception systémique, homogène, générale et abstraite des normes s'appliquant à une société d'individus inscrits dans les frontières d'un État (voir Donald Davis, vol. 1, p. 88). La critique de cette idéologie et de ses catégories est parfaitement salutaire et nécessaire, mais ce n'est pas en faisant comme si le concept avait été kidnappé par l'Occident et dépouillé de toutes les potentialités analytiques d'un concept universel qu'on pourra la mener à bien. Tout au contraire, c'est en insistant sur le caractère situé, local, historique et spécifique de la notion de droit que l'on pourra faire valoir la possibilité de penser autrement la normativité. Dire qu'il existe une nature ontologique du droit prenant des formes variées suppose qu'il y ait des éléments essentiels qui en soient constitutifs in abstracto, mais la question se pose alors de savoir lesquels. Sans doute vaudrait-il mieux prendre le langage « émique » au sérieux et trouver les comparaisons et traductions heuristiques, comme le recommandent Ludwig Wittgenstein ou Robin Collingwood. Parler de dharma (Donald Davis, vol. 1 et 2), de shariah (Judith Scheele, vol. 1, Morgan Clarke, vol. 2), de coutume (Paul Dresch ou Paul Brand, vol. 1), de nomos ou de ius (Georgy Kantor, vol. 1) en termes de droit, c'est commettre une extrapolation abusive. Dire que ce concept universel résiste indûment à

6. Dont l'attestation empirique est, qui plus est, problématique, comme chez Judith Scheele (vol. 2), qui parle de juridisme, s'agissant du Nord du Tchad, à partir de récits à propos de règles et non d'actions soutenues par des règles, ou chez Elizabeth Ewart (vol. 2), qui, dans le contexte amazonien, parle d'absence de règles, esthétiques ou morales par exemple, mais néglige le fait que le défaut de formulation de règles ne signifie pas l'absence de celles-ci. 
l'extrapolation, et ce pour cause d'ethnocentrisme (voir l'introduction de Paul Dresch, vol. 1), c'est confondre l'objet de la critique et la nature de cet objet. II y a une faille logique dans ce raisonnement : si l'anthropologie contemporaine a du mal à voir du droit en dehors d'un pouvoir commun à obliger à l'obéissance (voir l'introduction de Hannah Skoda, vol. 1), c'est sans doute parce qu'il n'y a pas d'autre façon d'envisager ce concept situé ; et ce n'est pas parce qu'on peut légitimement adresser au droit la critique de son association au pouvoir (Donald Davis, vol. 1, p. 88) que le droit n'est pas ce que vise cette critique. Que gagne-t-on, d'ailleurs, concrètement, à vouloir étendre l'usage du concept de droit urbi et orbi ? Est-il doté d'une vertu si particulière que ne pas y assimiler quelque chose présentant une ressemblance doive être vécu comme une tare ou un opprobre ? Si la charia est «le chemin de Dieu à travers la vie » (Judith Scheele, vol. 1, p. 201), pourquoi faudrait-il donc que ce soit un droit ? C'est une erreur de catégorie que de prendre un concept spécifique pour un concept générique, analytique et universel, et c'est une inversion logique que de lui reprocher la conception ethnocentrique qui serait la sienne et de lui donner une acception extensive, faisant disparaître au passage toute son historicité et sa contextualité. Sans parler de l'erreur nominaliste qui consiste à considérer que, parce qu'on utilise un même terme pour parler de «droit romain » ou de « droit islamique », il s'agit donc bien de droit (Judith Scheele, vol. 1, p. 199), alors précisément que c'est l'imposition des catégories cognitives spécifiques d'un contexte particulier à un objet d'un autre contexte, également particulier, qui a conduit à utiliser ce même terme.

\section{- L'auteur}

Directeur de recherche au CNRS, Baudouin Dupret est membre du Centre d'étude des mouvements sociaux (CEMS) à l'École des hautes études en sociales. Ses recherches portent sur l'approche théorique, sociologique et anthropologique de la norme et du droit (particulièrement dans les sociétés arabes et islamiques).

Parmi ses publications récentes : La Charia. Des sources à la pratique, un concept pluriel (La Découverte, 2014); Law At Work: Studies in Legal Ethnomethods (coédité avec M. Lynch et T. Berard) (Oxford University Press, 2015) ; Practices of Truth: An Ethnomethodological Inquiry Into Arab Contexts (Benjamins, 2011) ; Adjudication in Action: An Ethnomethodology of Law, Morality and Justice (Ashgate, 2011). 